\title{
A COMPARATIVE STUDY OF BIOACTIVE MOLECULES IN TREATMENT AND CONTROL OF CANCER
}

\section{MUKESH CHANDER}

Dean Research (Former) \& Assistant Professor, Bioprocess Lab, P.G. Department of Biotechnology, Khalsa College

(Autonomous), Amritsar - 143 003, India

\begin{abstract}
Bioactive molecules are obtained from living organisms and have variety of properties including anticancer, ant proliferative, anti-Parkinson, anti-Alzheimer etc. Out of these, cancer has emerged as an epidemic especially in the developing and third world countries. The cancer has been described as an uncontrolled cell growth leading to formation of carcinoma, nodes, lumps or hyperplasia conditions. The causative agents for this mutated cell conditions have been defined as "Risk Groups /factors" which may lead to cancer. Till date the traditional radiation therapy alone or coupled with chemotherapy has been practised as sole treatment of this disease. However, the survival rate of treated patients has been found to be very low. The present study describes role of various bioactive molecules in control of cancer. An effort has been made to ensemble the large data of bioactive compounds of microbial, plant, animal, food / herb / spices origin for their anticancer activity in minimum space possible in the present review.

KEYWORDS: Anticancer Metabolites, Antiproliferative Compounds, Bioactive Molecules, Microbes, Herbal Anticancer Compounds
\end{abstract}

Received: Jun 08, 2020; Accepted: Jun 28, 2020; Published: Sep 01, 2020; Paper Id.: IJMPERDJUN20201004

\section{INTRODUCTION}

The bioactive compounds are extra nutritional constituent and micro constituents of foods, studied intensively for health benefits. The results of many epidemiologic studies have shown protective effects of bioactive compounds of plant-based diets on cancer. These compounds vary widely in chemical structure, function and hence are grouped accordingly. Phenolic compounds, including their subcategory, flavonoids, are present in all plants and have been studied extensively in cereals, legumes, nuts, olive oil, vegetables, fruits, tea, and red wine. Many phenolic compounds have antioxidant properties, and some studies have demonstrated favourable effects on thrombosis and tumour control. Although some epidemiologic studies have reported protective associations between flavonoids or other phenolics and CVD and cancer, other studies have not found these associations. Various phytoestrogens are present in soy, flaxseed oil, whole grains, fruits, and vegetables have shown favourable effects on other CVD risk factors, in cell culture models of cancer. However, because phytoestrogens act both as partial estrogen agonists and antagonists, their effects on cancer are likely complex. Hydroxytyrosol, one of many phenolics in olives and olive oil, is a potent antioxidant. Resveratrol, found in nuts and red wine, has antioxidant, antithrombotic, and antiinflammatory properties, and inhibits carcinogenesis. Lycopene, a potent antioxidant carotenoid in tomatoes and other fruits, is thought to protect against prostate and other cancers, and inhibits tumour cell growth in animals. Organosulfur compounds in garlic and onions, isothiocyanates in cruciferous vegetables, and monoterpenes in citrus fruits, cherries, and herbs have anticarcinogenicand cardio-protective properties. In summary, numerous bioactive compounds appear to have beneficial health effects. From a practical perspective, this translates to 
recommending a diet rich in a variety of fruits, vegetables, whole grains, legumes, oils, and nuts[1]. The anticancer properties of these biochemicals have been discussed in the coming text.

Cancer can be activated by environmental carcinogens, inflammatory agents, and tumour promoters by modulating transcription factors, anti-apoptotic proteins, pro-apoptotic proteins, protein kinases, cell cycle proteins, cell adhesion molecules, and growth signalling pathways Some of the most critical pathways include the NF-B pathway, receptor tyrosine kinase receptor [(RTK, epidermal growth factor receptor (EGFR)] pathways, the mitogen-activated protein kinase (MAPK) pathways and regulation of the cell cycle.

The activator protein-1 (AP-1) pathway is also linked to cancerous growth and regulation of gulation, cell transformation, apoptosis, cellular proliferation, repression of tumour -suppressor genes, as well as involvement in the stages of tumour metastasis. Coriander and fennel have been found to decrease expression of both MAPK and c-Jun Nterminal kinase (JNK), which is another component of MAPK pathways.

Herbs, spices, and their bioactive components can inhibit, and sometimes induce pathways that regulate cell division, cell proliferation, detoxification. Clarification is also needed regarding which types of cells respond to various herbs, spices, and their bioactive components.

\section{UNICELLULAR ORGANISMS}

\section{Cyanobacteria}

The blue green algae appeared approximately 3.5 billion years old. The cyanobacteria are prokaryotic algae that exist as unicellular species or as colony forming units. Being autotrophic, it constitute the primary first level organisms in food chains in water ecosystems. Moreover, these prokaryotes play a significant role in balancing nitrogen $(\mathrm{N})$ and $\mathrm{CO}_{2}$ interrelation in the biosphere.

A few algal species produce cyanobacterial cyclopeptides; having a pharmacophore structure that may lead to development of a novel class of anticancer therapeutics with activity against chemotherapy-refractory metastatic cancers. Microcystins are synthesized by a non ribosomal enzyme complexas peptides, encoded by the microcystin (mcy) gene cluster. Mcy includes genes for peptide synthetases, polyketide synthases, mixed peptide synthetases and tailoring enzymes. Their ecological role and function is currently related to numerous effects on phytoplankton and zooplankton. MCs have evolved to function as a defense mechanism of cyanobacteria against grazing.

\section{MULTICELLULAR ORGANISMS}

Multi-cellular organisms are composed of variety of cells influenced by adaptive evolutionary principles during the course of our individual lives. Following are examples to illustrate the application of basic evolutionary principles

\section{Humoral Immunity}

The immune response concerns the reaction of the body (self) to invasion by foreign substances (non-self). In case of humoral immunity, foreign substances (living) known as antigens stimulate B-lymphocyte cells to produce molecules known as antibodies. Antibodies react with antigens to tag them for further immunological responses. A specific antibody, carried by a B-lymphocyte, is capable of "recognizing" (by chemical binding) a limited range of antigenic molecular shapes. This combination of signals stimulates the particular B-lymphocyte to divide (mitosis) and make daughters of 
itself. The proportion of these particular B-cells increase in the lymphocyte population, which then, create more of the antibody. Some B-lymphocytes become factories for the production of large numbers of antibodies to fight the current infection. Adaptive immune system is advantageous in a world where we are confronted with rapidly evolving pathogens and parasites.

\section{Cancer}

In multi-cellular organisms, various specialized cells are genetically identical in most of parts such as liver, kidney. Kidney cells differ from liver cells with respect to differential patterns of gene activation. The formation of a cancer cell is typically a multi-step process in which several mutant alleles must be acquired to produce a mutant.

\section{ANIMAL SOURCES}

\section{Colostrum}

Colostrum is the first food that a mammal produces for her young before she produces milk. It is filled with immuneboosting properties that protect and strengthen the newborn's health and has curative properties for cancer. Colostrum is rich in immunoglobins, antimicrobial peptides and others bioactive molecules, including growth factors.Natural bioactive substances in it are nucleosides and nucleotides [22]. Two factors present in colostrum are TGF-beta and EGF. Colostrum has following app. for cancer treatment:

- Cancer cells randomly exist even within normal healthy people. The difference is that their immunity works well to kill off the cancer cells long before they grow and spread throughout the body.

- Colostrum has transforming growth factor (TGF) polypeptides which effectively fight cancer cells. Colostrum also contains Lactoferrin, which helps to regulate iron metabolism. Lactoferrin is a milk protein that binds and transports iron throughout the body. It also supports the body's immunity through its inhibition of pathogens and its antiviral and antibacterial properties. It stops or kills pathogens by blocking their iron absorption.

- Colostrum also contains phytic acid and Alpha Lactalbumin. Phytic acid is an antioxidant that also blocks iron from the cancer cells similar to Lactoferrin activities. Alpha Lactalbumin stops cancer cells from growing.

- The colostrum cure holds promise in helping to restore the immune response against the growth of harmful, cancerous cells so that the body is able for self-cure.

\section{Milk}

Milk forms a rich source of biologically important/active components, especially the milk nprotein, perofrming performing many kinds of biological functions. In this review we focus on antibacterial and antiviral properties of milk proteins and milk protein derivatives. The latter include chemically modified proteins and enzymatically induced peptides. If such peptides are released by enzymes present within the digestive tract (e.g. trypsin or pepsin), they play a role in the health defense system. The antibacterial proteins are lactoferrin, lactoperoxidase, and lysozyme. Furthermore, antibacterial peptides originating from caseins and whey proteins. Antiviral effects of milk proteins and derivatives thereof. Special focus is directed to the antiviral action towards the human immunodeficiency virus (HIV) and the human cytomegalovirus (HCMV). Unmodified milk proteins are generally not active against these viruses. An exception is lactoferrin, which shows significant antiviral activity against both HIV and cancer. Milk proteins provide excellent nutrition. Milk proteins 
can also exert numerous physiological activities. These activities include enhancement of immune function, defense against pathogenic bacteria, viruses, and yeasts, and development of the gut and its functions. Besides the naturally occurring, biologically active proteins present in milk, a variety of bioactive peptides are encrypted within the sequence of milk proteins that are released upon suitable hydrolysis of the precursor protein.

A large range of bioactivities has been reported for milk protein components, with some showing more than one kind of biological activity. The most important antimicrobial and antitumour peptides derived from milk proteins, especially those that may have a physiological significance. Special attention is given to the generation of these peptides by the action of different proteolytic enzymes. The milk whey proteins include alpha-lactalbumin, beta-lactoglobulin and many growth factors showing anti-inflammatory and prohealing effects.

\section{PLANT SOURCES}

A major area of interest for the use of biologically active chemical components of plants, i.e. phytochemicals, is for cancer prevention. For example, several phytochemicals have been shown to reduce colonic aberrant crypt foci formation caused by administration of the carcinogen azoxymethane to rats. The list of potential anticarcinogenic phytochemicals under study is long, but includes dithiolthiones, glucosinolates and isothiocyanates (cruciferous vegetables), coumarins and limonene (citrus fruits), isoflavones inositol hexaphosphate, protease inhibitors and saponins (soybean), carotenoids (palm oil and yellow vegetables) and allium compounds (onion, garlic and leek).The major focus of phytochemical research has been on cancer prevention, Prebiotics (including chicory roots) and symbiotics.

Prebiotics are a collective term for non-digestible but fermentable dietary carbohydrates that may selectively stimulate growth of certain bacterial groups resident in the colon, such as lactobacilli and eu bacteria, considered to be beneficial for the human host. Digestion-resistant short-chain carbohydrates are also referred to as non-digestible oligosaccharides or low-digestible carbohydrates. There is much interest currently in the commercial sector in adding prebiotic supplements, particularly fructo-oligosaccharides and soybean oligosaccharides, to food products to influence colonic flora, i.e. 'bifidogenic' effects. These may be useful in prevention of intestinal infections and modulation of the intestinal immune response in celiac or inflammatory bowel disease. An example of a prebiotic is Chicory fructooligosaccharides (ChiFos). Following ingestion, ChiFos reach the colon intact and acts as a prebiotic with bifidogenic effects. The potential use of ChiFos as sugar substitutes and fat replacers makes them of particular interest to the food industry.

\section{Aloe Vera}

Aloe vera has been widely used as a medicine and additive in many cosmetic products, contains a wide range of potentially bioactive molecules e.g. It contains several potentially bioactive salicylates, magnesium lactate, lupeol, campestrol, sitosterol, linolenic acid, aloctin A and anthraquinones. It is currently available as leaf exudate and gel. Aloe vera possesses beneficial effects for gastrointestinal disease, including anti-inflammatory, analgesic and pro-healing effects. In addition, a component of Aloe vera, has been reported to accelerate healing and reduce pain in aphthous stomatitis and prevent stressinduced gastric ulceration in rats.

\section{Soyabean}

Soya foods are the predominant source of dietary isoflavones and are also a rich source of trypsin inhibitor, saponins and sphingolipids, all of which have potential health benefits, including cancer prevention (breast, prostate and colon) and 
regulation of the host immune system. Soya protein is currently used as part of milk feed substitutes for infants with cows' milk intolerance.

Soyabean is an Anti Cancer Diet. Soyfoods help protect against several types of cancer, including lung, colon, rectal, stomach, and prostate cancer. Soyfoods are rich in compounds called phytochemicals. One particular family of phytochemicals, isoflavones, may fight cancer in a variety of ways. Isoflavones are found in significant amounts only in soybeans and in soyfoods, such as soy milk, and textured soy protein. Genistein is a chemical compound found only in soy in our daily foods. Genistein blocks an angiogenesis, the growth of new blood vessels that nourish malignant tumours.Genistein may also work against cancers that depend on hormones to grow, such as breast and prostate cancer. Genistein may interfere with these hormones, thus inhibiting the development of cancer cells and tumours. Important implications for treatment of solid tumours, including malignancies of the breast, prostate, colon, and brain. The beneficial effects of isoflavones include:

- A decrease in blood androgen levels by increasing the level of (SHBG) sex-hormone binding globulin, which binds testosterone, lowering its availability for cancer cells.

- Inhibition of alpha-5 reductase, and other enzymes that converts testosterone to its most potent form hence restricting cancer cells growth

- Inhibition of tumour blood vessel formation.

- Decrease in insulin growth factor-1 (IGF-1), which may be a marker for increased prostate cancer risk. Researchers believe that cancer risk increase may be in part due to the change from a diet high in isoflavones to which is low in isoflavones, lower in fruits and vegetables and higher in total fat.

Prostate cancer cell growth was inhibited only in the cells treated with genistein into animal models. These animals ate either a soy-free diet or a soy-based diet. The progression of prostate cancer was reduced by $25 \%$ in the animals on the soy-based diet [4].

Some Prostate Cancer Recommendations:

- Soy protein intake should be 35 to 40 grams per day.

- A good way to add soy protein to your diet is by having a soy-protein smoothie with breakfast.

- Start increasing your soy intake gradually.

- Consumption of isoflavone-rich foods was associated with a decreased risk of breast canceramong postmenopausal women.

\section{Citrus Fruits}

Citrus fruits are one of the most important and largest fruit crops produced worldwide. This type of fruit is most commonly consumed as fresh produce and juice. It is known for high phyto nutrient content that contributes to its health benefits. Flavonoids, one of the major classes of compounds in citrus have antioxidant, anti-tumour, anti-inflammatory activities. The most abundant flavonoids found in citrus are hesperidin, neohesperidin, rutin, neoponcirin, and nobiletin. The citrus carotenoid intake may decrease the risk of cardiovascular disease and cancer. Among the carotenoids, $\beta$-carotene, lutein, 
zeaxanthin, $\beta$-cryptoxanthin, and lycopene are found to be the most in citrus fruits. Citrus limonoids, have ntioxidant, hypocholesterolemic, antiviral, insecticidal, and anticancer activities [1].

\section{Flavonoids}

Flavonoids are water-soluble secondary metabolites and provide much of the flavor and color to fruits and vegetables. There are six major subclasses of flavonoids: flavones, flavonols, flavanones, flavanols, and isoflavones. In humans, flavonoids have a wide range of bioactivities including anticancer, anti-inflammatory properties. One class of flavonoids is the Apigenin, has anti-inflammatory and anticarcinogenic effects. The second class of flavonoids is the flavonols, which are commonly present in many fruits vegetables. One flavonol of great interest due to its anticancer properties is quercetin. Quercetinhas to be a free-radical scavenging antioxidant, as well as having the ability to induce apoptosis and block different phases of the cell-cycle in a variety of cancer cell lines[4].

Curcumin is a fat-soluble polyphenolic compound, bioactive compound of interest curing many types of cancer including breast, prostate, oral. The curcumin is so widely studied in a variety of cancers, is because of its many pharmacological properties including antioxidant, anti-inflammatory, antimicrobial, antitumour activitiesin vitro[3].

\section{HERBS AND SPICES}

Historically herbs and spices have enjoyed a rich tradition of use for their flavor-enhancement characteristics and for their medicinal properties. The rising prevalence of chronic diseases world-wide and the corresponding rise in health care costs is propelling interest among researchers and the public for these food related items for multiple health benefits, including a reduction in cancer risk and modification of tumour behavior. A growing body of epidemiological and preclinical evidence points to culinary herbs and spices as minor dietary constituents with multiple anticancer characteristics. This review focuses on the anti-microbial, antioxidant, and anti-tumourigenic properties of herbs and spices, their ability to influence carcinogen bioactivation, and likely anticancer contributions. While culinary herbs and spices present intriguing possibilities for health promotion, more complete information is needed about the actual exposures to dietary components that are needed to bring about a response and the molecular targets for specific herbs and spices.

The use of spices and herbs contributes to conflicts in scientific literature about the precise role of diet and cancer prevention where herbs and spices were not specifically addressed in the analyses they did concluded other dietary protective factors may exist and contribute to individual variations in risk. Herbs and spices used to season and preserve food may also contribute to eating behaviors. For instance, one of the highest rates of gastric cancer mortality in Europe occurs in Italy. Over 1,200 gastric cancer patients and more than 1,100 controls from 7 areas grouped into high and low risk areas, pointed to several categories of foods associated with gastric cancer risk.NCI's cancer Biomedical Informatics $\operatorname{Grid}^{\mathrm{TM}}\left(\mathrm{caBIG}{ }^{\mathrm{TM}}\right)$, an open source, open access information network which is connecting the cancer research community and enabling the sharing of data and tools, recently funded the development of a nutrition ontology tool which integrates into the NCI Thesaurus[17].

Concentrations of herbs and spices used in food preparation often vary based on individual preference, but the concentration of herbs and spices in finished foods frequently falls within the range of $0.5-1 \%$. A recent attempt to evaluate human methyl eugenol exposure for the National Toxicology Program (NTP), U.S. Department of Health and Human Services and in partnership with the National Cancer Institute (NCI), highlights many of the challenges associated with exposure assessment for herbs and spices in general. Methyl eugenol belongs to a family of chemicals which includes 
several naturally occurring compounds commonly found in the human diet, such as isoeugenol, eugenol, estragole, and safrole, which can be found in spices such as nutmeg and all spice, herbs such as basil and tarragon, in addition to fruits such as bananas and oranges.A popular herb in China, extracts of oldenlandia have been part of treating viral infections, snake bites, cancers of the colon, stomach and oesophagus. A tonic of cleavers has been used for shrinking tumours and lymphatic drainage, especially with patients whose cancers have reached the lymph nodes. In raw form, it can also be added to soups.

\section{SPHINGOLIPIDS}

Sphingolipids are sphingosine-based lipid molecules that have important functions in cellular signal transduction and in a variety of cellular processes including proliferation, differentiation, programmed cell death (apoptosis). Ceramides, dihydroceramide, sphingosine and sphingosine-1-phosphate are examples of those bioactive sphingolipids. They have a major impact on determination of the cell fate by contributing to the cell survival or cell death through apoptosis. Despite the number of carbon atoms in the fatty acid chain changes the physiological role; ceramides generally exert suppressive roles on the cell proliferation. There have been several enzymes identified, that are responsible for the conversion of ceramide into other sphingolipid derivatives. Sphingosine-1-phosphate is an example of such sphingolipid derivatives which has anti-apoptotic effects. As they have significant impacts particularly on the cell death and survival, bioactive sphingolipids have a great potential to be targets in cancer therapy [4,5]. An antibody treat many of the deadliest solid and liquid tumour s. This unique monoclonal antibody, called Sphingomab ${ }^{\mathrm{TM}}$, was tested in several animal models of human cancer and was shown to significantly retard cancer growth on a consistent basis; in some cases, it eliminated the tumour altogether [6].

A monoclonal antibody against sphingosine-1-phosphate (S1P) and has validated S1P as a therapeutic target for cancer. The most deadly, multi-resistant cancers-including lung, breast, melanoma, and ovarian cancers-responded well to the Sphingomab ${ }^{\mathrm{TM}}$ approach of targeting S1P. The Sphingomab ${ }^{\mathrm{TM}}$ treatment not only blocked the effects of S1P on the cancer cells themselves, but also prevented tumour angiogenesis, which is the formation of new blood vessels that feed the growing tumour $[7,8]$.

Inhibitors of glucosylceramide synthase (GCS) may be useful in preventing chemotherapy resistance. For example, combinations of fenretinide (4-HPR), which is known to elevate ceramide and (dihydro)ceramide levels with the inhibitors of GCS or SK, such as PPMP (1-phenyl-2-palmitoylamino-3-morpholino- 1-propanol) or safingol (L-threodihydrosphingosine), were reported to suppress the growth of various human cancer cells, synergistically.

A network of metabolic enzymes regulates the generation of ceramide and S1P, and these enzymes serve as transducers of sphingolipid-mediated responses that are coupled to various exogenous or endogenous cellular signals. Consistent with their key roles in the regulation of cancer growth and therapy, attenuation of ceramide generation and/or increased S1P levels are implicated in the development of resistance to drug-induced apoptosis, and escape from cell death. These data strongly suggest that advances in the molecular and biochemical understanding of sphingolipid metabolism and function will lead to the development of novel therapeutic strategies against human cancers, which may also help overcome drug resistance[9].

Ceramide mediates the regulation of growth arrest, senescence, and apoptosis. Some of these biological functions might be controlled through novel sphingolipid-protein interactions [10]. Most frequently, these direct targets of ceramide 
constitute protein phosphatises and kinases that regulate important phosphatis pathways in cancer, such as protein kinase $\mathrm{C}$ (PKC), MAP kinases, or phospholipase D. The regulation of protein hosphatise-1 and -2 (PP1 and PP2A)-family enzymes, also referred to as ceramide activated protein phosphatises (CAPPs), by ceramide has been well documented previously [11-14].

Sphingolipids have emerged as bioeffector molecules, which control various aspects of cell growth, proliferation, and anti-cancer therapeutics $[15,16]$.

\section{ANTIBIOTICS}

Antibiotics are drugs that are used to treat infections caused by bacteria and other organisms, including protozoa, parasites, and fungi. Many treatments for cancer destroy disease-fighting white blood cells, thereby reducing the body's ability to fight infection. For example, bladder, pulmonary, and urinary tract infections may occur with chemotherapy. Single-celled organisms called protozoa are rarely a problem for healthy individuals. However, they can cause serious infections in individuals with low white blood cell counts. Because of the dangers that infections present for cancer patients, antibiotic treatment often is initiated before the exact nature of the infection has been determined; instead, the choice of antibiotic may depend on the site of the infection and the organism that is likely to be the cause.

\section{Treatments and Prophylaxis}

Decisions concerning treatments depend largely on the kind of cancer and the stage to which the cancer has progressed. Because cancer cells invade many parts of the body simultaneously, a combination of targeted therapies are necessary to fight this disease. Chemotherapy or radiation destroys cells, leaving the body unable to fight off infection. Surgical procedures involve removing tumours or injecting patients with non-cancerous cells, or transplants[17-19, 38].

Cancer patients undergoing surgery often require the use of antibiotics prior to surgery to prevent infection. Without antibiotics, bacteria during surgery to remove tumour s could invade the bloodstream and infect other areas of the body. The common antibiotics that are used during cancer treatment include:

- Atovaquone (Mapren): Antiprotozoal drug used to prevent and treat a very serious type of pneumonia called Pneumocystis carinii pneumonia (PCP), in individuals who experience serious side effects with SMZ-TMP (Sulfamethoxazole/Trimethoprim, brand name Bactrim).

- Aztreonam (Azactam): monobactam antibiotic used to treat gram-negative bacterial infections of the urinary and lower respiratory tracts and the female organs.

- Cefepime (Maxipime), ceftazidime (Ceptaz, Fortaz, Tazicef, Tazidime), and ceftriaxone sodium (Rocephin): members of a group of antibiotics called cephalosporins treat infections of the urinary and lower respiratory tracts.

- Cefepime: $500 \mathrm{mg}$ to $2 \mathrm{gm}$, injected into a vein or muscle, every 8-12 hours for 7-10 days.

- Ceftazidime: $250 \mathrm{mg}$ to $2 \mathrm{gm}$, injected into a vein or muscle, every 8-12 hours.

- Ceftriaxone: 1-2 gm, injected into a vein or muscle, every 24 hours.

- Ciprofloxacin: 500-750 mg of the tablet or suspension. 
- Clindamycin: $150-300 \mathrm{mg}$ of capsule or solution, every six hours; 300-600 mg every six to eight hours or $900 \mathrm{mg}$ every eight hours, injected into a vein or muscle.

- Gentamicin: dosage determined by body weight, every 8-24 hours for at least 7-10 days, injected into a vein or muscle.

- Pentamidine: for treating PCP, $4 \mathrm{mg}$ per $\mathrm{kg}$ (1.8 mg per lb) of body weight, once per day for 14-21 days, injected into a vein over one to two hours, while lying down.

- Pyrimethamine: for toxoplasmosis, 25-200 mg tablets, taken with other medicine, for several weeks[20].

\section{MICRO RNA}

MicroRNAs are short ribonucleic acid (RNA) molecules, on average only 22 nucleotides long and are found in all eukaryotic cells. miRNAs are post-transcriptional regulators that bind to complementary sequences on target messenger RNA transcripts (mRNAs), usually resulting in translational repression and gene silencing. The human genome may encode over 1000 miRNAs, which may target about $60 \%$ of mammalian genes and are abundant in many human cell types. By the early 1990s, miRNAs were not recognized as a distinct class of biologic regulators with conserved functions. Since then, miRNA research has revealed multiple roles in negative regulation (transcript degradation and sequestering, translational suppression) and possible involvement in positive regulation (transcriptional and translational activation)[13, $20]$.

\section{Synthesis}

Most micro RNA genes are found in intergenic regions or in anti-sense orientation to genes and contain their own miRNA gene promoter and regulatory units. As much as $40 \%$ of miRNA genes may lie in the introns of protein and non-protein coding genes or even in exons.

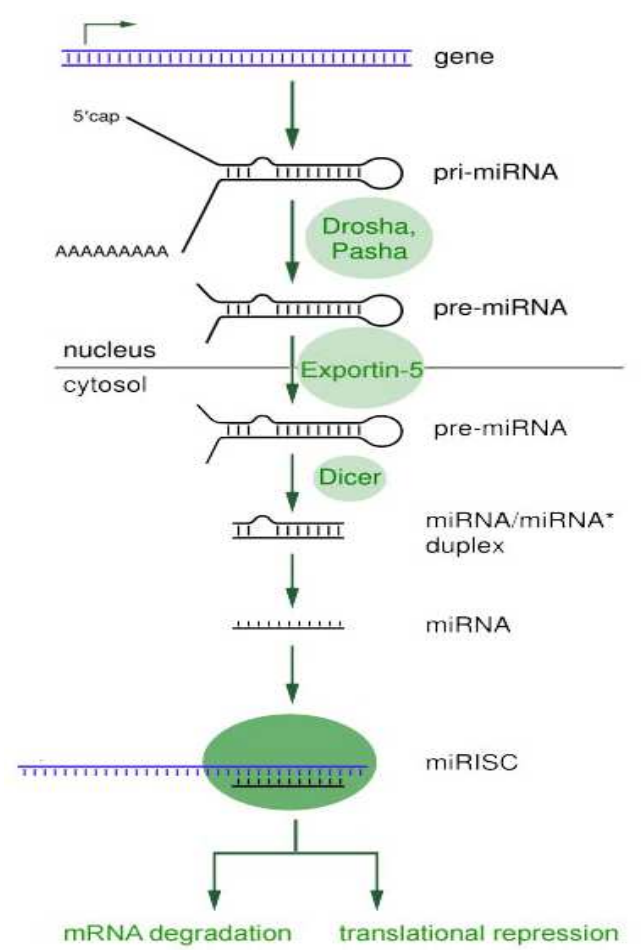

Figure 1: Micro-RNA s are Produced from their Own Genes or from Introns. 
The DNA template is not the final word on mature miRNA production: $6 \%$ of human miRNAs show RNA editing, the site-specific modification of RNA sequences to yield products different from those encoded by their DNA. This increases the diversity and scope of miRNA action beyond that implicated from the genome alone.

\section{Transcription}

miRNA genes are usually transcribed by RNA polymerase II. The polymerase often binds to a promoter found near the DNA sequence encoding what will become the hairpin loop of the pre-miRNA. The resulting transcript is capped with a specially-modified nucleotide at the 5' end, polyadenylated with multiple adenosines (a poly(A) tail), and spliced. The product, called a primary miRNA (pri-miRNA), may be hundreds or thousands of nucleotides in length and contain one or more miRNA stem loops. When a stem loop precursor is found in the 3' UTR, a transcript may serve as a pri-miRNA and a mRNA. RNA polymerase III (Pol III) transcribes some miRNAs, especially those with upstream Alu sequences, transfer RNAs (tRNAs), and mammalian wide interspersed repeat (MWIR) promoter units.

\section{Nuclear Processing}

A singlepri-miRNA may contain from one to six miRNA precursors. These hairpin loop structures are composed of about 70 nucleotides each [21,22]. Each hairpin is flanked by sequences necessary for efficient processing. The double-stranded RNA structure of the hairpins in a pre-miRNA is recognized by a nuclear protein known as DiGeorge Syndrome Critical Region 8 (DGCR8 or "Pasha" in invertebrates), named for its association with DiGeorge Syndrome. DGCR8 associates with the enzyme Drosha, a protein that cuts RNA, to form the "Microprocessor" complex. In this complex, DGCR8 orients the catalytic RNase III domain of Drosha to liberate hairpins from pri-miRNAs by cleaving RNA about eleven nucleotides from the hairpin base (two helical RNA turns into the stem). The resulting hairpin, known as a pre-miRNA (precursormiRNA), has a two-nucleotide overhang at its 3' end; it has 3' hydroxyl and 5' phosphate groups [33].

\section{Nuclear Export}

Pre-miRNA hairpins are exported from the nucleus in process involving the nucleocytoplasmic shuttleExportin-5. This protein, a member of the karyopherin family, recognizes a two-nucleotide overhang left by the RNase III enzyme Drosha at the 3' end of the pre-miRNA hairpin. Exportin-5-mediated transport to the cytoplasm is energy-dependent, using GTP bound to the Ran protein.

\section{Cytoplasmic Processing}

In the cytoplasm, the pre-miRNA hairpin is cleaved by the RNase III enzyme Dicer. This endoribonuclease interacts with the 3' end of the hairpin and cuts away the loop joining the 3' and 5' arms, yielding an imperfect miRNA: miRNA duplex about 22 nucleotides in length. Overall hairpin length and loop size influence the efficiency of Dicer processing, and the imperfect nature of the miRNA:miRNA pairing also affects cleavage. Although either strand of the duplex may potentially act as a functional miRNA, only one strand is incorporated into RNA-induced silencing complex (RISC) [12].

\section{ARACHIDONIC ACID}

It is a polyunsaturated omega- 6 fatty acid. It is the counterpart to the saturated arachidonic acid found in peanut oil. Arachidonic acid is a carboxylic acid with a 20-carbon chain and four cisdouble bonds; the first double bond is located at the sixth carbon from the omega ends $[23,24]$. Arachidonic acid is a polyunsaturated fatty acid that is present in the phospholipids of membranes of the body's cells, and is abundant in the brain, muscles, liver. 


\section{Synthesis}

Arachidonic acid is freed from a phospholipid molecule by the enzyme phospholipase A2 ( $\left.\mathrm{PLA}_{2}\right)$, which cleaves off the fatty acid, but can also be generated from DAG by diacylglycerol lipase [35-37]. Arachidonic acid generated for signaling purposes appears to be derived by the action of a phosphatidylcholine-specific cytosolic phospholipase A2 (cPLA 2,85 $\mathrm{kDa}$ ), whereas inflammatory arachidonic acid is generated by the action of a low-molecular-weight secretory PLA 2 $\left(\mathrm{sPLA}_{2}, 14-18 \mathrm{kDa}\right)$. Arachidonic acid is a precursor in the production of eicosanoids:

- The enzymes cyclooxygenase and peroxidase lead to prostaglandin $\mathrm{H} 2$, which in turn is used to produce the prostaglandins, prostacyclin, and thromboxanes.

- The enzyme 5-lipoxygenase leads to 5-HPETE, which in turn is used to produce the leukotrienes.

- Arachidonic acid is also used in the biosynthesis of anandamide.

- Some arachidonic acid is converted into hydroxyeicosatetraenoic acids (HETEs) and epoxyeicosatrienoic acids (EETs) by epoxygenase.

The production of these derivatives and their action in the body are collectively known as the arachidonic acid cascade.

\section{Role in Cancer Treatment}

Most of the effects of arachidonic acid are attributable to its conversion by oxygenases to prostaglandins, leukotrienes, and other bioactive products [25-28]. Arachidonic acid bioactivity is an undue reliance on oxygenase inhibitors in invoking a biological role for the supposed "products" of oxygenase metabolism. In fact, it is not uncommon to find cell lines that do not metabolize added arachidonic acid and that are essentially lacking in the ability to generate oxygenase metabolites [2934]. There are specific G-protein-coupled receptors for lipids such as lysophosphatidic acid and platelet-activating factor, and the bio actions of ester are said to mimic the role of natural diglycerides. Similarly, there are specific receptors for the esters and amides of arachidonate, namely the cannabinoid receptors for 2-arachidonylglycerol and arachidonylethanolamide.

\section{MICROALGAL DOCOSAHEXAENOIC ACID}

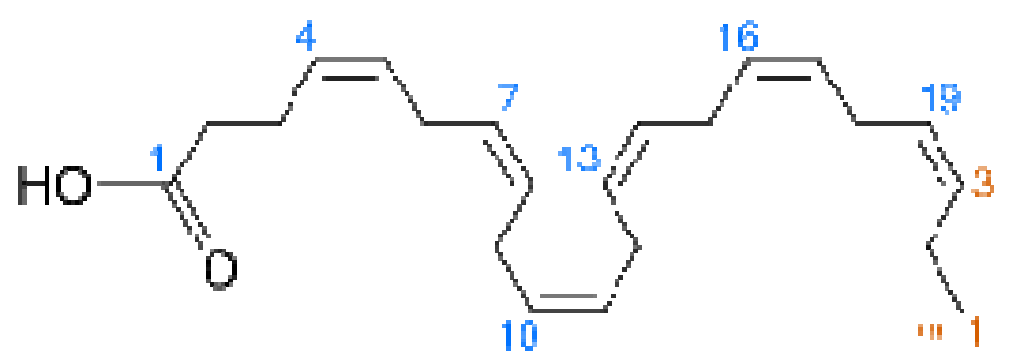

Figure 2: Structure of DHA.

Docosahexaenoic acid (DHA) is an omega-3 fatty acid. In chemical structure, DHA is a carboxylic acid with a ${ }^{22-}$ $\mathrm{C}$ chain[38] and six $c$ is double bonds; the first double bond is located at the third carbon from the omega end. Its trivial name is cervonic acid, its systematic name is all-cis-docosa-4,7,10,13,16,19-hexa-enoic acid, and its shorthand name is 22:6(n-3) in the nomenclature of fatty acids. Cold-water oceanic fish oils are rich in DHA. Most of the DHA in fish and 
complex organisms with access to cold-water oceanic foods originates in photosynthetic and heterotrophic microalgae, and becomes increasingly concentrated in organisms, as they move up the food chain $[29,40]$. DHA is also commercially manufactured from microalgae; Crypthecodiniumcohnii and another of the genus Schizochytrium.DHA manufactured using microalgae is vegetarian. Some animals with access to seafood make very little DHA through metabolism, but obtain it in the diet [41,42].

\section{NANO PARTICLES}

Recent successful medical trials of a cancer treatment involving the use of nanotechnology may open up important new avenues for the diagnosis and treatment of other cancers and diseases [43-45]. A nanometre is one billionth of a metre or about the size of 10 hydrogen atoms. The techniques range from various chemical and biological processes used to "construct" structures-in some cases atom by atom-to the etching methods used to produce computer chips.

BrachySil $^{\circledR}$ is a tiny structure about one-millionth of a metre in size and made up of modified particles of silicon impregnated with the radioactive isotope of phosphorus 32P. Unlike other radiation treatments that involve focussing beams of radiation on tumours, BrachySil ${ }^{\circledR}$ is injected directly into the cancer using a fine gauge needle. By using 32P, the radiation is limited to a range of just 8 millimetres, resulting in the killing of tumour cells rather than healthy tissue. For several years, doctors have been using a similar technique known as brachytherapy-injecting radioisotopes directly into tumours. The difficulty was that the injected material would not remain in the cancer, but would over time be carried to other parts of the body. The advantage of $\mathrm{BrachySil}^{\circledR}$ is that its silicon structures, while small, prevent the radioisotope from leaking away.

Hence, it is proposed that the cancer induced by various type of pollutants [45, 46], carcinogens [47] and mutagenic agents may cause substantial damage to human life, however the bioactive molecules may find their role in prevention $[13,45,48]$ and containment of the disease. However, this study need further verification in terms of concentration, dosage and duration of intake of these bioactive molecules.

\section{REFERENCES}

1. Wang, Y., Chuang, Y., Ku, Y., Quantitation of bioactive compounds in citrus fruits cultivated in Taiwan. Food Chem.2007; 102(4): 1163-1171.

2. Patil, J., Jayaprakasha, G., Murthy, K., Chetti, M., Patil, B., Characterization of Citrus aurantifolia bioactive compounds and their inhibition of pancreatic cancer cells through apoptosis. Microchem. J.2010; 57(22):10933-42.

3. Ujiki, M., Ding, X., Salabat, M., Bentrem, D.et al., Apigenin inhibits pancreatic cancer cell proliferation through G2/M cell cycle arrest. Mol. Cancer.2006; 5: 76. doi: 10.1186/1476-4598-5-76.

4. Liu, H., Jiang, W., Xie, M., Flavonoids: recent advances as anticancer drugs. Recent Pat Anticancer Drug Discov. 2010; 5(2): 152-64.

5. Schatzkin, A., Kelloff, G., Chemo- and dietary prevention of colorectal cancer. Eur J Cancer.2006; 31A (7-8): 1198-204.

6. Abe A, Radin NS, Shayman JA, Wotring LL, Zipkin RE, Sivakumar R, Ruggieri JM, Carson KG, Ganem B. Structural and stereochemical studies of potent inhibitors of glucosylceramide synthase and tumour cell growth.J Lipid Res. 1995;36(3):611-21. 
7. Akao Y, Banno Y, Nakagawa Y, Hasegawa N, Kim TJ, Murate T, Igarashi Y, Nozawa Y. High expression of sphingosine kinase 1 and SIP receptors in chemotherapy-resistant prostate cancer PC3 cells and their camptothecin-induced upregulation. BiochemBiophys Res Commun. 2006; 342(4):1284-90.

8. Alexander S, Min J, Alexander H. Dictyosteliumdiscoideum to human cells: pharmacogenetic studies demonstrate a role for sphingolipids in chemoresistance. BiochimBiophysActa. 2006; 1760 (3): $301-9$.

9. Andrieu-Abadie N, Levade T. Sphingomyelin hydrolysis during apoptosis. BiochimBiophysActa. 2002; 30;1585(2-3):126-34.

10. Argraves KM, Wilkerson BA, Argraves WS. Sphingosine-1-phosphate signaling in vasculogenesis and angiogenesis. World J Biol Chem. 2010; 26; 1(10): 291-97.

11. Baran Y, Salas A, Senkal CE, Gunduz U, Bielawski J, Obeid LM, Ogretmen B. Alterations of ceramide/sphingosine 1phosphate rheostat involved in the regulation of resistance to imatinib-induced apoptosis in K562 human chronic myeloid leukemia cells. J Biol Chem. 2007; 13; 282 (15):10922-34.

12. Sardi E, Tyihak E. Simple determination of formaldehyde in dimedoneadduct form in biological samples by high performance liquid chromatography.Biomed. Chromatogr. 1994; 8 (6); 313-14.

13. Tyihák E, Trézl L, Szende B. In: In Stress of Life, From molecules to Man. P Csermely., editor. Annals of the New York Academy of Sciences. 1998.1

14. Wie H, Zhang R, Wang C, Zheng H, LiA, Chou KC, Wie DQ. J. Theor. Biol. 2007; 21; 244(4):692-702.

15. Tyagi N, Moshal KS, Ovechkin AV, Rodriquez W, Steed M, Henderson B, Roberts AM, Joshua TG, Tyagi SC. Cystathionine beta synthase gene dose dependent vascular remodeling in murine model of hyperhomocysteinemia. Int $\mathrm{J}$ PhysiolPathophysiolPharmacol. 2011; 30; 3(3): 210-22.

16. Baylin SB, Herman JG, Graff JR, Vertino PM, Issa JP. Alterations in DNA methylation: a fundamental aspect of neoplasia. Adv. Cancer Res. 1998;72:141-96.

17. Huszti Z, Tyihák E. Formation of formaldehyde from S-adenosyl-L-[methyl-'H]methionine transmethylation of histamine. FEBS Lett. 1986;209:362.

18. Pereira LAR, Todorova M, Cai X, Makaroff C, Emery R, Moffatt S. Methyl recycling activities are co-ordinately regulated during plant development. J. Exp. Bot. 2007; 58:1083-1098.

19. Glycoproteomic analysis of two mouse mammary cell lines during transforming growth factor (TGF)-beta induced epithelial to mesenchymal transition". 7thspace.com. 2009-01-08.

20. Daopin S, Piez K, Ogawa Y, Davies D. Crystal structure of transforming growth factor-beta 2: an unusual fold for the superfamily". Science. 1992;257 (5068); 369-73.

21. Hoff, M. H. and Dix, T. A. Redox-mediated activation of latent transforming growth factor-beta 1. Mol. Endocrinol. 1996; 10,1077-1083

22. Blaise F, Paik W.K. In: Protein Methylation. Paik W.K, Kim S, editors. Boca Raton, Florida: CRC Press Inc; 1990. pp. 59-65.

23. Baylin SB, Ohm JE. Epigenetic gene silencing in cancer - a mechanism for early oncogenic pathway addiction? Nat. Rev. Cancer. 2006;6:107.

24. Chen RZ, Pettersson U, Beard C, Jackson-Grusby L, Jaenisch R. DNA hypomethylation leads to elevated mutation rates. Nature. 1998; 395 (6697): 89-93.

25. Duerre JA, Lee CT. In vivo methylation and turnover of rat brain histones. J Neurochem. 23: 541-47. 
26. Teas J. The dietary intake of Laminaria, a brown seaweed, and breast cancer prevention. Nutr Cancer. 1983; 4: $217-22$.

27. Reddy B S, Cohen L A, McCoy G D, Hill P, Weisburger J H, Wynder E L. Nutrition and its relationship to cancer. Adv Cancer Res. 1980; 32: 237-345.

28. Wynder E L, Kajitani T, Kuno J, Lucas J C J, Depalo A, Farrow J. A comparison of survival rates between American and Japanese patients with breast cancer. SurgGynecol Obstet. 1963; 117:196-200.

29. Morrison A S, Black M M, Lowe C R, MacMahon B, Yuasa S. Some international differences in histology and survival in breast cancer. International Journal of Cancer. 1973; 11:261-7.

30. Kanemori M, Prygrocki M. Results of breast conservation therapy from a single-institution community hospital in Hawaii with a predominantly Japanese population. Int J RadiatOncolBiol Phys. 2005; 62(1):193-7.

31. Pineda M D, White E, Kristal A R, Taylor V. Asian breast cancer survival in the US: a comparison between Asian immigrants, US-born Asian Americans and Caucasians. Int J Epidemiol. 2001; 30(5):976-82

32. Ziegler R G, Hoover R N, Pike M C, et al. Migration patterns and breast cancer risk in Asian-American women. J Natl Cancer Inst. 1993; 85:1819-27.

33. Thompson $L U$, Chen J M, Li T, Strasser-Weippi K, Goss P E. Dietary flaxseed alters tumour biological markers in postmenopausal breast cancer. Clin Cancer Res. 2005; 11(10):3828-35.

34. Belay A. The potential application of Spirulina(Arthrospira) as a nutritional and therapeutic supplement in health management. J Am Nutraceut Assoc. 2002; 5(2):27-48.

35. Abdulqader G, Barsanti L, Tredici M R. Harvest of Arthrospiraplatensis from Lake Kossorom (Chad) and its household usage among the Kanembu. Journal of Applies Phycology. 2000; 12(3-5):493-8.

36. Wu L C, Ho J A, Shieh M C, Lu I W. Antioxidant and antiproliferativeactivities of Spirulina and Chlorella water extracts. J Agric Food Chem. 2005; 53(10):4207-12.

37. Subhashini J, Mahipal S V, Reddy M C, Mallikarjuna Reddy M, Rachamallu A, Reddanna P. Molecular mechanisms in CPhycocyanin induced apoptosis in human chronic myeloid leukemia cell line-K562. BiochemPharmacol. 2004; 68(3):453-62

38. Reddy M C, Subhashini J, Mahipal S V, et al. C-Phycocyanin, a selective cyclooxygenase-2 inhibitor, induces apoptosis in lipopolysaccharide-stimulated RAW 264.7 macrophages. BiochemBiophys Res Commun 2003; 304(2):385-92.

39. Liu X M, Zhang HQ. Effect of polysaccharide from Spirulinaplatensis on hematopoietic cells proliferation, apoptosis and Bcl2 expression in mice bearing tumour treated with chemotherapy. Yao XueXueBao. 2002; 37(8):616-20

40. Chen F, Zhang Q. Inhibitive effects of spirulina on aberrant crypts in colon induced by dimethylhydrazine. Zhonghua 1995; 29(1):13-7.

41. Martek Biosciences Corporation (2007). "History of Martek". Archived from the original on February 5, 2007.

42. Meharban Singh (2005). "Essential Fatty Acids, DHA and the Human Brain from the Indian Journal of Pediatrics, Volume 72" (PDF). http://medind.nic.in/icb/t05/i3/icbt05i3p239.pdf.

43. Youdim KA, Martin A, Joseph JA (2000). "Essential fatty acids and the brain: possible health implications.http://www.ncbi.nlm.nih.gov/sites/entrez?cmd=Retrieve $\& d b=$ pubmed\&dopt=AbstractPlus\&list_uids=10817922.

44. http://www.springerlink.com/content/3547007r16268860/.

45. Lukiw WJ, Cui JG, Marcheselli VL, Bodker M, Botkjaer A, Gotlinger K, Serhan CN, Bazan NG. "A role for docosahexaenoic acid-derived neuroprotectin D1 in neural cell survival and Alzheimer disease". J Clin Invest.115 (10): 2774-83. 
46. Chander M (2018). “Anticancer Efficacy of Some Plant Phenolics - A Recent Scenario”.IntJ CurrMicrobiol App Sci. 7 (10): 1746-68. ISSN: 2319-7706. doi: 10.20546/ijcmas.2018.710.200.

47. Chander M, Arora D S (2014). "Biodegradation of a Dye by Different White-rot Fungi on a Novel Agro Residue Based Medium”.Lignocellulose.3 (1): 37-50. ISSN: 2252-0287.

48. Chander M, Kaur I. (2015). “An Industrial Dye Decolourisation by Phlebia sp.”IntJ CurrMicrobiol App Sci. 4 (05). ISSN: 2319-7706.

49. Devi, P. R., \& Singh, K. I. (2016).Efficacy of new molecules, spinosad and monocrotophos on the incidence of Cnaphalocrocismedinalis Guenee under kharif rice crop ecosystem of Manipur valley. Int. J. Agric. Sci. Res, 6(1), 7-14.

50. Maryvijaya, T., \& Palavesam, A. Effect of Temperature on Biomolecules in Penaeus Indicus.

51. Gupta, A., Naraniwal, M., \& Kothari, V. (2012).Modern extraction methods for preparation of bioactive plant extracts. International journal of applied and natural sciences, 1(1), 8-26.

52. Chethan, K., Hanumanthaswamy, B., \& Nagaraja, R. Evaluation of Insecticide Molecules Against Turmeric Shoot Borer, Conogethes Punctiferalis Gueene (Lepidoptera: Pyralidae). 

